\title{
Comparison of Helmet Therapy and Counter Positioning for Deformational Plagiocephaly
}

\author{
Se Yon Kim, $\mathrm{MD}^{1}$, Moon-Sung Park, $\mathrm{MD}^{2}$, Jeong-In Yang, $\mathrm{MD}^{3}$, Shin-Young Yim, $\mathrm{MD}^{1}$ \\ ${ }^{1}$ The Clinic for Torticollis, Department of Physical Medicine and Rehabilitation, \\ Departments of ${ }^{2}$ Pediatrics, ${ }^{3}$ Obstetrics and Gynecology, Ajou University School of Medicine, Suwon, Korea
}

Objective To compare effectiveness on correcting cranial and ear asymmetry between helmet therapy and counter positioning for deformational plagiocephaly (DP).

Methods Retrospective data of children diagnosed with DP who visited our clinic from November 2010 to October 2012 were reviewed. Subjects $\leq 10$ months of age who showed $\geq 10 \mathrm{~mm}$ of diagonal difference were included for analysis. For DP treatment, information on both helmet therapy and counter positioning was given and either of the two was chosen by each family. Head circumference, cranial asymmetry measurements including diagonal difference, cranial vault asymmetry index, radial symmetry index, and ear shift were obtained by 3-dimensional head-surface laser scan at the time of initiation and termination of therapy.

Results Twenty-seven subjects were included: 21 had helmet therapy and 6 underwent counter positioning. There was no significant difference of baseline characteristics, head circumferences and cranial asymmetry measurements at the initiation of therapy. The mean duration of therapy was $4.30 \pm 1.27$ months in the helmet therapy group and $4.08 \pm 0.95$ months in the counter positioning group $(p=0.770)$. While cranial asymmetry measurements improved in both groups, significantly more improvement was observed with helmet therapy. There was no significant difference of the head circumference growth between the two groups at the end of therapy.

Conclusion Helmet therapy resulted in more favorable outcomes in correcting cranial and ear asymmetry than counter positioning on moderate to severe DP without compromising head growth.

Keywords Deformational plagiocephaly, Nonsynostotic plagiocephaly, Patient positioning, Cephalometry, Facial asymmetry

Received May 28, 2013; Accepted August 6, 2013

Corresponding author: Shin-Young Yim

The Clinic for Torticollis, Department of Physical Medicine and Rehabilitation, Ajou University School of Medicine, 206 Worldcup-ro, Yeongtong-gu, Suwon 443-721, Korea

Tel: +82-31-219-5284, Fax: +82-31-219-5209, E-mail: syyim@ajou.ac.kr

(c) This is an open-access article distributed under the terms of the Creative Commons Attribution Non-Commercial License (http://creativecommons. org/licenses/by-nc/3.0) which permits unrestricted noncommercial use, distribution, and reproduction in any medium, provided the original work is properly cited.

Copyright $\odot 2013$ by Korean Academy of Rehabilitation Medicine

\section{INTRODUCTION}

Plagiocephaly literally means "oblique head," combining the Greek words plagios, meaning oblique, and kephale, meaning head [1]. The term can be used to describe asymmetric head shapes resulting from both synostotic and nonsynostotic causes. Deformational plagiocephaly (DP) arises from repeated external pressure to the same area of the flexible cranium during the fetal or neonatal periods [2]. When this repeated pressure is 
applied to one side of the occiput, it results in ipsilateral occipital flattening, frontal bone protrusion, and anterior shifting of the ipsilateral ear [3].

The incidence of DP has risen since 1992, when the American Academy of Pediatrics started its Back to Sleep campaign, encouraging parents to place infants on their backs to decrease the incidence of Sudden Infant Death Syndrome [4]. The incidence of DP was only $0.3 \%$ in the 1970s [5], but the current reported estimates since 1992 range from $2 \%$ [6] to $47 \%$ [7], depending on the diagnostic criteria used and the age of the children involved [6-11]. Despite the fact that the American Academy of Pediatrics released recommendations to prevent DP in 2003 [12], the incidence of DP remains high [6,9-11].

Clarren et al. [13] introduced helmet therapy for children with DP in 1979. The first helmet was made of fiberglass and modeled on a plaster cast of the subjects' head. Four children wore these helmets, and their cranial asymmetry improved without any serious complications. Helmets used for DP treatment are currently custommade for each individual. They are constructed to apply full-contact external pressure to the protruding area and to allow space where bony expansion is desired $[2,14]$. In 1998, the Food and Drug Administration of the United States approved the cranial orthosis as a class II medical device, intended for infants between 3 and 18 months of age with moderate to severe DP.

The current treatment for patients with moderate to severe DP consists of either helmet therapy or counter positioning, with reports supporting the efficacy of both techniques $[10,15,16]$, although with varying results on comparing the efficacy between them [17-22]. A study conducted in 70 DP patients reported a greater improvement in the diagonal difference (DD) measurement with helmet therapy than with counter positioning, but the same improvement was not seen in the cranial vault asymmetry index (CVAI) [21]. A longitudinal study of 298 DP patients revealed a more favorable correction outcome with helmet therapy than counter positioning, although helmet therapy had a longer treatment time [22]. There are no definable standard criteria for starting helmet therapy and treatment decision is influenced more strongly by factors other than medical evidence. One study reported that physician preference exists between specialties, and suggested that neurosurgeons are less likely to prescribe helmet therapy for DP than plastic surgeons [23].

The therapeutic effect of both helmet therapy and counter positioning on ear shift remains inconclusive, with few results reported. Kluba et al. [24] reported the effects of helmet therapy on pre-existing ear shift in patients with DP, implying that helmet therapy has a corrective effect. However, the same study showed that ear shift can arise de novo during helmet therapy in patients who did not have ear shift at the beginning of treatment.

Although helmet therapy is widely used and studied in the United States, few studies have reported on the effectiveness of helmet therapy in Asian or, in particular, Korean children because helmet therapy is not widely performed in Korea [25]. The aim of this study was to assess the efficacy of helmet therapy and compare it with counter positioning for correcting cranial asymmetry and ear shift in Korean children with moderate to severe DP.

\section{MATERIALS AND METHODS}

In this retrospective study, we analyzed the medical records of patients diagnosed and treated for DP at our clinic, part of a tertiary medical center in Korea, between November 2010 and October 2012.

\section{Subjects}

All children with a diagnosis of DP were evaluated for study inclusion. DP was defined as nonsynostotic unilateral occipital flattening. The diagnosis of DP was confirmed by 3-dimensional (3D) computed tomography, performed to exclude the diagnosis of craniosynostotic plagiocephaly. Children with known neurodevelopmental disorders, such as cerebral palsy, autistic spectrum disorders, genetic aberrations, and metabolic diseases, were excluded.

Included patients had a DD $>10 \mathrm{~mm}$ and were $\leq 10$ months of age. According to the protocol at our clinic, the caregivers of patients meeting these criteria were given information about both helmet therapy and counter positioning; the treatment decision was made by the caregivers. Patients treated with helmet therapy were designated the helmet therapy group and patients treated with counter positioning were designated the counter positioning group. 


\section{Helmet therapy}

In the helmet therapy group, a custom-molded helmet was made for each child based on their initial 3D headsurface laser scan (STARscanner laser data acquisition system; Orthomerica Products, Orlando, FL, USA). The helmet (Orthokorea, Seoul, Korea) consisted of a polyethylene foam liner and a copolymer outer shell. Caregivers were told to keep the child in the helmet as much as possible, with $>23$ hours of daily use recommended. After the initial helmet fitting, follow-up laser scans were performed every 3-4 weeks to adjust for both head growth and skull-shape change. Helmet wearing time was que-
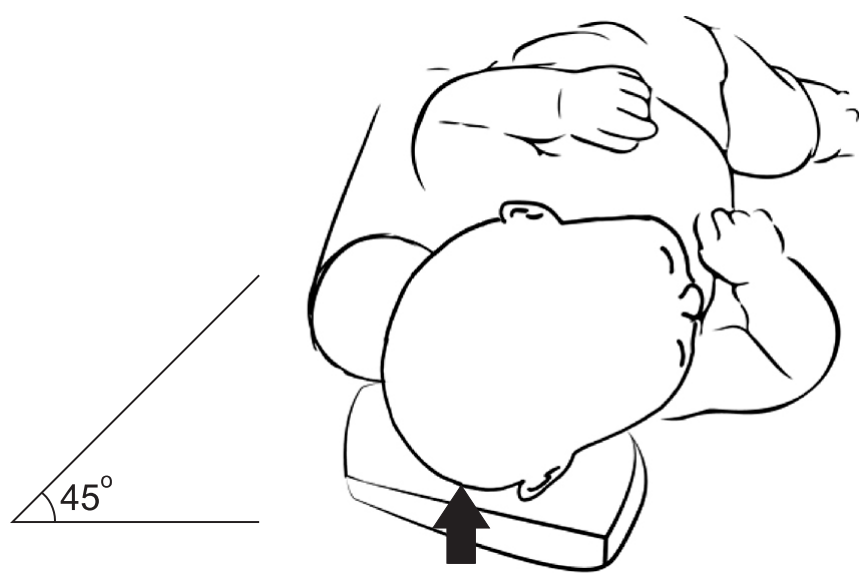

Fig. 1. The head was to be positioned in a 45-degree turn, resting against a hard surface on the nonflattened side of occiput when the child was lying on its back.

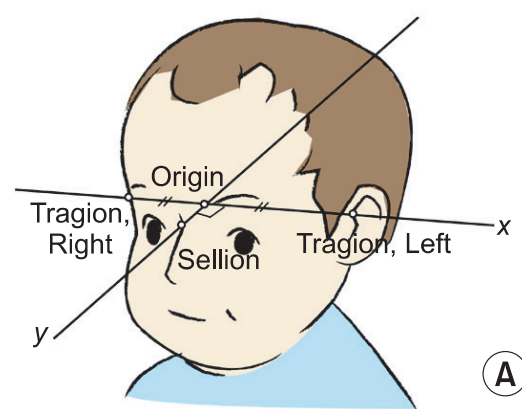

ried every follow-up visit for laser scanning and helmet wearing time $<20$ hours per day was considered therapy failure and was not included in this study. Helmet therapy was terminated when DD $<6 \mathrm{~mm}[25]$ and CVAI $<3.5 \%$ [18] and/or no further helmet adjustment was possible due to cranial growth.

\section{Counter positioning therapy}

In the counter positioning group, the following program was recommended: 1) When the child was lying on its back, the head was to be positioned in a 45-degree turn, resting against a hard surface on the nonflattened side of occiput (Fig. 1). As much time as possible, with a minimum of 12 hours per day, was to be spent in counter positioning. 2) The crib and other environments were to be rearranged to encourage counter positioning. 3) A firm pillow was to be used for resting the nonflattened side, to achieve adequate pressure on the prominent portion of the head.

Caregivers were interviewed monthly by telephone to ensure compliance and to repeat instructions. Caregivers were told to follow these instructions for a minimum of 3 months, at that time, patients were reassessed with a second laser scan.

\section{Anthropometric measurements}

Anthropometric measurements were obtained at the initiation and termination timepoints of each therapy, using the 3D head-surface laser scanning device. The fol-

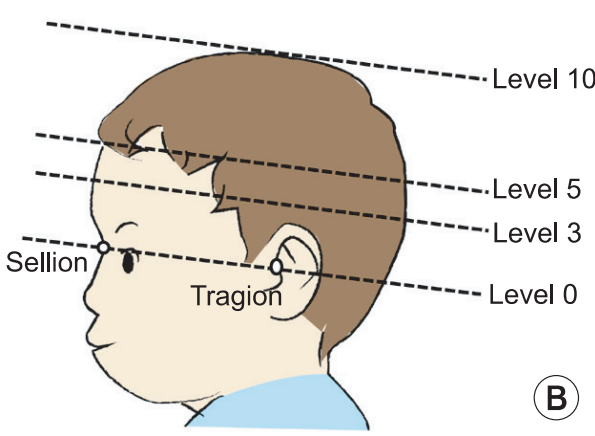

Fig. 2. Pictures showing anatomical landmarks (A) and the reference plane (B). Sellion is set at the most concave point in the soft tissue on the naso-frontal angle between the forehead slope and the proximal nasal bridge. Tragion is set at the upper margin of the tragus. After setting these landmarks the origin is set at the midpoint between the right and left tragia. Then the $\mathrm{Y}$-axis is defined as a line through the sellion and the origin. The X-axis was defined as the line perpendicular to the Y-axis that crosses the origin. Level 0 is the reference cross-sectional plane including sellion and both tragia. The portion of the cranium superior to the reference plane was divided into 9 equally spaced crosssectional planes, each parallel to the reference plane, where the level 10 is the plane through vertex. Anthropometric measurements on level 3 and 5 planes were used in this study. 
lowing landmarks were set for anthropometric measurement (Fig. 2A):

- Tragion: the point at the upper margin of the tragus,

- Origin: the midpoint between the right and left tragia,

- Sellion: the most concave point in the soft tissue on the nasofrontal suture angle (between the forehead slope and the proximal nasal bridge),

- Y-axis: the line connecting the sellion and the origin,

- X-axis: the line perpendicular to the Y-axis that crosses the origin.

A cross-sectional plane through the sellion and both tragia was set as the reference plane and designated the level 0 plane. The plane parallel to the level 0 plane that passed through the vertex of the head was designated the level 10 plane. The portion of the cranium superior to the reference/level 0 plane was divided into nine equally spaced cross-sectional planes, each parallel to the reference plane (Fig. 2B). The following anthropometric measurements were obtained at levels 3 and 5 (Fig. 3):

- DD ( $\mathrm{mm}$ ): the difference between two diagonal cranial diameters, 30 degrees from the Y-axis [26]; $\mathrm{DD}=$ longer diagonal-shorter diagonal

- CVAI (\%): DD/short cranial diagonal $\times 100$ [18]

- Radial symmetry index (RSI) (mm): Starting at the front of the head, vector lengths were measured at 15-degree intervals and summed for each side of the head. The absolute value of the difference between the right-sided sum and the left-sided sum was the RSI. A RSI of $0 \mathrm{~mm}$ indicated perfect symmetry [27].

- Ear shift (mm): the anteroposterior distance between the right and left tragia, perpendicular to the $\mathrm{X}$-axis at the level 0 plane [26]. Ear shift was measured twice by a single blinded observer, using Adobe Acrobat Pro (Adobe Systems, San Jose, CA, USA). No significant difference was found between the two measurements; the mean value was used for this study.

\section{Statistical analyses}

Statistical Package for the Social Sciences (SPSS) ver. 21.0 (IBM Corp., Armonk, NY, USA) was used for statistical analysis. The statistical significance of the results was tested using the nonparametric Mann-Whitney U-test. The chi-square test was used for comparing dichotomous variables such as patient sex and the occipital flattening side. Statistical significance was set at a p-value of $<0.05$. To ensure that this study has appropriate level of statistical power, post hoc statistical power analysis was done using G*Power ver. 3.1 (http://www.psycho.uni-duesseldorf.de/abteilungen/aap/gpower3) [28,29].

\section{RESULTS}

\section{Baseline patient characteristics}

The helmet therapy group consisted of 21 patients, with 6 patients in the counter positioning group. The baseline patient characteristics are presented in Table 1. The
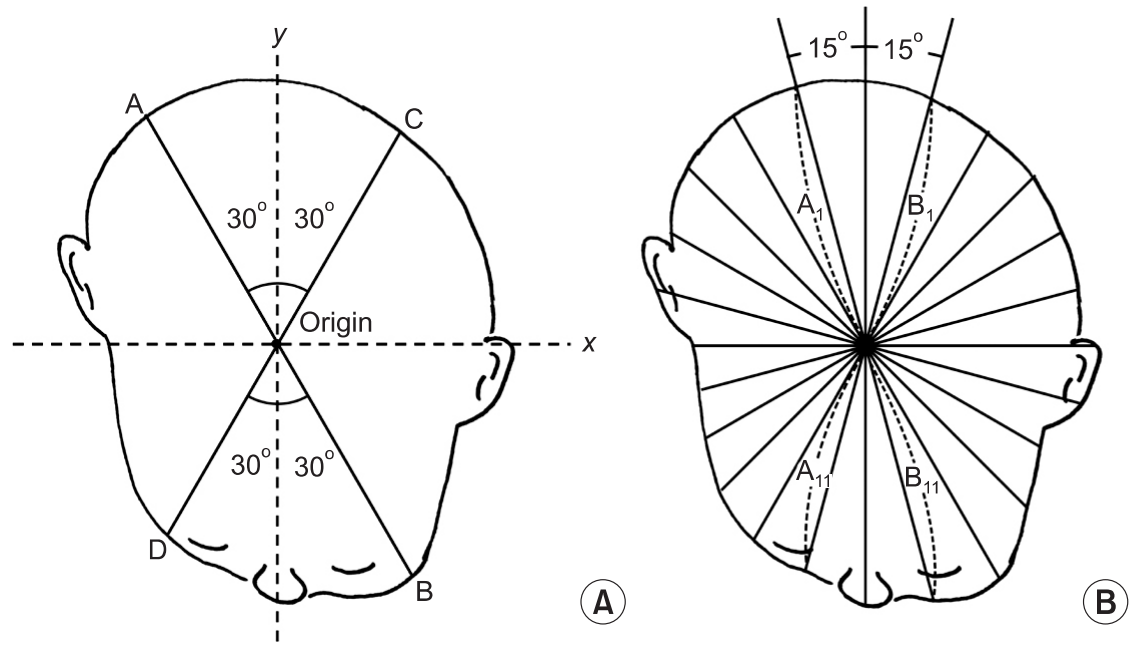

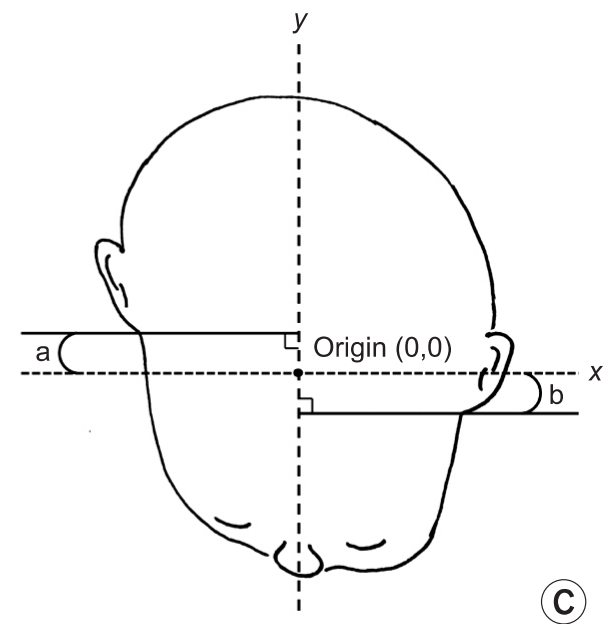

Fig. 3. (A) Diagonal difference (DD; $m m$ )=longer diagonal (AB)-shorter diagonal (CD). Cranial vault asymmetry index (\%) $=\mathrm{DD} / \mathrm{CD} \times 100$. (B) Radial symmetry index $(\mathrm{mm})=\left|\left(\mathrm{A}_{1}+\mathrm{A}_{2}+\ldots+\mathrm{A}_{11}\right)-\left(\mathrm{B}_{1}+\mathrm{B}_{2}+\ldots+\mathrm{B}_{11}\right)\right| \cdot(\mathrm{C})$ The ear shift $(\mathrm{mm})=|\mathrm{a}-\mathrm{b}|$. 
Table 1. Characteristics of subjects

\begin{tabular}{|c|c|c|c|}
\hline Characteristic & $\begin{array}{l}\text { Helmet therapy group } \\
\qquad(n=21)\end{array}$ & $\begin{array}{c}\text { Counter positioning } \\
(\mathbf{n}=6)\end{array}$ & p-value \\
\hline Age (mo) & $5.62 \pm 2.06$ & $5.60 \pm 2.04$ & 0.884 \\
\hline Boy:Girl & $16: 5$ & $4: 2$ & 0.639 \\
\hline Duration of therapy (mo) & $4.30 \pm 1.27$ & $4.08 \pm 0.95$ & 0.770 \\
\hline Occipital flattening (right:left) & $16: 5$ & $5: 1$ & 0.716 \\
\hline \multicolumn{4}{|l|}{ Head circumference (mm) } \\
\hline Level 3 & $431.39 \pm 17.34$ & $433.03 \pm 20.49$ & 0.816 \\
\hline Level 5 & $417.85 \pm 17.94$ & $421.05 \pm 18.82$ & 0.484 \\
\hline \multicolumn{4}{|l|}{ Diagonal difference $(\mathrm{mm})$} \\
\hline Level 3 & $13.28 \pm 3.57$ & $11.38 \pm 3.30$ & 0.220 \\
\hline Level 5 & $14.94 \pm 3.60$ & $13.75 \pm 4.28$ & 0.560 \\
\hline \multicolumn{4}{|l|}{ Cranial vault asymmetry index (\%) } \\
\hline Level 3 & $9.95 \pm 2.68$ & $8.54 \pm 2.90$ & 0.180 \\
\hline Level 5 & $11.76 \pm 2.89$ & $10.86 \pm 3.89$ & 0.600 \\
\hline \multicolumn{4}{|l|}{ Radial asymmetry index (mm) } \\
\hline Level 3 & $56.29 \pm 12.72$ & $51.93 \pm 15.27$ & 0.448 \\
\hline Level 5 & $66.20 \pm 17.01$ & $66.00 \pm 20.83$ & 0.953 \\
\hline Ear shift (mm) & $5.67 \pm 2.39$ & $6.09 \pm 3.53$ & 0.899 \\
\hline
\end{tabular}

Values are presented as mean \pm standard deviation or numbers.

mean patient age in the helmet therapy group was 5.62 months, compared with 5.60 months in the counter positioning group $(\mathrm{p}=0.884)$. The mean duration of therapy was 4.30 months in the helmet therapy group and 4.08 months in the counter positioning group $(\mathrm{p}=0.770)$. The groups were similar in makeup regarding patient sex and the side of occipital flattening. There was no significant difference in the initial head circumference, the degree of cranial asymmetry, or the ear shift measurements before the initiation of therapy.

Change of anthropometric measurements during the study period in each group

Cranial asymmetry measurements, such as DD, CVAI, and RSI, and ear shift measurements showed significant improvement during the study period in the helmet group (Table 2). Comparison of all the asymmetry measurements from the initiation to the conclusion of treatment period showed a statistically significant $p$-value of $<0.001$. However, in the counter positioning group, cranial asymmetry measurements, such as DD, CVAI, and RSI, showed no significant difference from the initiation and to the conclusion of treatment period (Table 3). Only ear shift showed significant improvement during the treat- ment period in the counter positioning group $(\mathrm{p}=0.026)$.

\section{Comparison of the anthropometric measurement} changes between groups

Comparison of the change in anthropometric measurements (DD, CVAI, RSI) at the level 3 and level 5 planes during the treatment period revealed a significantly better improvement in cranial asymmetry in the helmet therapy group than the counter positioning group (Table 4). The mean change in DD at level 3 was $-6.80 \mathrm{~mm}$ in the helmet therapy group $-1.33 \mathrm{~mm}$ in the counter positioning group $(\mathrm{p}=0.003)$. The mean change in DD at level 5 was $-7.69 \mathrm{~mm}$ in the helmet therapy group and $-1.60 \mathrm{~mm}$ in the counter positioning group $(\mathrm{p}=0.001)$. The mean change in CVAI in helmet therapy group and counter positioning group was $-5.51 \%$ and $-1.54 \%$, respectively, at level $3(\mathrm{p}=0.004)$ and $-6.64 \%$ and $-2.01 \%$, respectively, at level $5(\mathrm{p}=0.002)$. The RSI showed significant greater change in the helmet therapy group compared with the counter positioning group $(-25.34 \mathrm{~mm}$ vs. $-9.18 \mathrm{~mm}$ at level $3 ;-29.97 \mathrm{~mm}$ vs. -10.48 $\mathrm{mm}$ at level 5). Ear shift also showed better outcome in the helmet therapy group, showing mean change of $-3.04 \mathrm{~mm}$ compared with $-1.09 \mathrm{~mm}$ in the counter positioning group during the treatment period $(\mathrm{p}=0.044)$. 
Table 2. Anthropometric measurements during treatment period in the helmet group

\begin{tabular}{|c|c|c|c|}
\hline Measurement & Initiation & Conclusion & p-value \\
\hline \multicolumn{4}{|l|}{ Head circumference (mm) } \\
\hline Level 3 & $431.39 \pm 17.34$ & $451.94 \pm 16.60$ & $0.001^{*}$ \\
\hline Level 5 & $417.85 \pm 17.94$ & $443.12 \pm 15.82$ & $0.001^{*}$ \\
\hline \multicolumn{4}{|l|}{ Diagonal difference $(\mathrm{mm})$} \\
\hline Level 3 & $13.28 \pm 3.57$ & $6.48 \pm 1.92$ & $<0.001^{*}$ \\
\hline Level 5 & $14.94 \pm 3.60$ & $7.25 \pm 2.49$ & $<0.001^{*}$ \\
\hline \multicolumn{4}{|c|}{ Cranial vault asymmetry index (\%) } \\
\hline Level 3 & $9.95 \pm 2.68$ & $4.45 \pm 1.29$ & $<0.001^{*}$ \\
\hline Level 5 & $11.76 \pm 2.89$ & $5.13 \pm 3.09$ & $<0.001^{*}$ \\
\hline \multicolumn{4}{|c|}{ Radial asymmetry index (mm) } \\
\hline Level 3 & $56.29 \pm 12.72$ & $30.95 \pm 7.13$ & $<0.001^{*}$ \\
\hline Level 5 & $66.20 \pm 17.01$ & $36.24 \pm 8.92$ & $<0.001^{*}$ \\
\hline Ear shift (mm) & $5.67 \pm 2.39$ & $1.96 \pm 1.37$ & $<0.001^{*}$ \\
\hline
\end{tabular}

Values are presented as mean \pm standard deviation.

${ }^{*} \mathrm{p}<0.05$.

Table 3. Anthropometric measurements during treatment period in the counter positioning group

\begin{tabular}{|c|c|c|c|}
\hline Measurement & Initiation & Conclusion & p-value \\
\hline \multicolumn{4}{|l|}{ Head circumference (mm) } \\
\hline Level 3 & $433.03 \pm 20.49$ & $455.30 \pm 15.46$ & $0.041^{*}$ \\
\hline Level 5 & $421.05 \pm 18.82$ & $444.47 \pm 13.70$ & $0.037^{*}$ \\
\hline \multicolumn{4}{|l|}{ Diagonal difference (mm) } \\
\hline Level 3 & $11.38 \pm 3.30$ & $10.05 \pm 1.43$ & 0.937 \\
\hline Level 5 & $13.75 \pm 4.28$ & $12.15 \pm 5.56$ & 0.485 \\
\hline \multicolumn{4}{|c|}{ Cranial vault asymmetry index (\%) } \\
\hline Level 3 & $8.54 \pm 2.90$ & $7.01 \pm 1.15$ & 0.394 \\
\hline Level 5 & $10.86 \pm 3.89$ & $8.85 \pm 4.53$ & 0.394 \\
\hline \multicolumn{4}{|c|}{ Radial asymmetry index (mm) } \\
\hline Level 3 & $51.93 \pm 15.27$ & $42.75 \pm 7.61$ & 0.240 \\
\hline Level 5 & $66.00 \pm 20.83$ & $55.52 \pm 15.34$ & 0.589 \\
\hline Ear shift $(\mathrm{mm})$ & $6.09 \pm 3.53$ & $4.24 \pm 2.53$ & $0.026^{*}$ \\
\hline
\end{tabular}

Values are presented as mean \pm standard deviation.

${ }^{*} \mathrm{p}<0.05$.

Severity subgroup analysis in helmet therapy group

Subgroup analysis to compare treatment efficacy based on DD severity and greater improvement was done; results are shown in Table 5. The helmet therapy group was divided into two subgroups based on DD: moderate DP group $(10 \mathrm{~mm}<\mathrm{DD}<16 \mathrm{~mm})$ and severe $\mathrm{DP}$ group $(\mathrm{DD} \geq 16$ $\mathrm{mm})$. Changes in anthropometric measurements were compared between these two subgroups. In this subgroup analysis, all cranial asymmetry measurements and ear shift showed greater improvement in the severe DP group. All these differences were statistically significant, except for RSI at level 5 , which showed a p-value of 0.051 .

\section{Head growth}

The head circumference enlarged in all patients during treatment. There was no significant difference in head circumference before therapy, with a mean value at level 3 of $431.39 \mathrm{~mm}$ in the helmet therapy group and 433.03 
Table 4. Change of anthropometric measurements between groups at the conclusion of therapy

\begin{tabular}{|c|c|c|c|c|}
\hline Measurement & $\begin{array}{l}\text { Helmet therapy group } \\
\qquad(\mathrm{n}=21)\end{array}$ & $\begin{array}{l}\text { Counter positioning } \\
\text { group }(n=6)\end{array}$ & p-value & $\begin{array}{c}\text { Statistical } \\
\text { power }\end{array}$ \\
\hline \multicolumn{5}{|l|}{ Head circumference (mm) } \\
\hline Level 3 & $20.55 \pm 6.09$ & $22.27 \pm 10.37$ & 0.932 & \\
\hline Level 5 & $25.28 \pm 7.20$ & $23.42 \pm 10.12$ & 0.629 & \\
\hline \multicolumn{5}{|l|}{ Diagonal difference $(\mathrm{mm})$} \\
\hline Level 3 & $-6.80 \pm 2.82$ & $-1.33 \pm 3.39$ & $0.003^{*}$ & 0.95 \\
\hline Level 5 & $-7.69 \pm 2.31$ & $-1.60 \pm 3.66$ & $0.001^{*}$ & 0.96 \\
\hline \multicolumn{5}{|c|}{ Cranial vault asymmetry index (\%) } \\
\hline Level 3 & $-5.51 \pm 2.20$ & $-1.54 \pm 2.79$ & $0.004^{*}$ & 0.94 \\
\hline Level 5 & $-6.64 \pm 1.98$ & $-2.01 \pm 3.15$ & $0.002^{*}$ & 0.97 \\
\hline \multicolumn{5}{|c|}{ Radial asymmetry index (mm) } \\
\hline Level 3 & $-25.34 \pm 12.93$ & $-9.18 \pm 13.44$ & $0.022^{*}$ & 0.96 \\
\hline Level 5 & $-29.97 \pm 14.84$ & $-10.48 \pm 12.59$ & $0.005^{*}$ & 0.89 \\
\hline Ear shift (mm) & $-3.04 \pm 3.92$ & $-1.09 \pm 5.41$ & $0.044^{*}$ & 0.74 \\
\hline
\end{tabular}

Values are presented as mean \pm standard deviation.

${ }^{*} \mathrm{p}<0.05$.

Table 5. Change of anthropometric measurements during treatment period according to severity of plagiocephaly in each group

\begin{tabular}{|c|c|c|c|c|c|c|}
\hline \multirow[b]{2}{*}{ Measurement } & \multicolumn{3}{|c|}{ Helmet therapy group } & \multicolumn{3}{|c|}{ Counter positioning group } \\
\hline & $\begin{array}{l}\text { Severe } \\
(n=11)\end{array}$ & $\begin{array}{c}\text { Moderate } \\
(n=10)\end{array}$ & p-value & $\begin{array}{l}\text { Severe } \\
(n=3)\end{array}$ & $\begin{array}{c}\text { Moderate } \\
(n=3)\end{array}$ & p-value \\
\hline \multicolumn{7}{|l|}{ Diagonal difference $(\mathrm{mm})$} \\
\hline Level 3 & $-8.87 \pm 2.03$ & $-6.67 \pm 3.23$ & $0.001^{*}$ & $-4.85 \pm 1.98$ & $0.65 \pm 0.47$ & 0.057 \\
\hline Level 5 & $-8.94 \pm 2.25$ & $-3.86 \pm 3.391$ & $0.006^{*}$ & $-6.31 \pm 1.50$ & $0.67 \pm 1.65$ & 0.200 \\
\hline \multicolumn{7}{|c|}{ Cranial vault asymmetry index (\%) } \\
\hline Level 3 & $-7.11 \pm 1.58$ & $-5.61 \pm 2.47$ & $0.001^{*}$ & $-4.02 \pm 1.62$ & $0.06 \pm 0.53$ & 0.057 \\
\hline Level 5 & $-7.70 \pm 1.86$ & $-4.06 \pm 3.09$ & $0.010^{*}$ & $-5.46 \pm 1.39$ & $0.05 \pm 1.54$ & 0.200 \\
\hline \multicolumn{7}{|c|}{ Radial asymmetry index (mm) } \\
\hline Level 3 & $-34.83 \pm 10.02$ & $-25.50 \pm 9.07$ & $0.001^{*}$ & $-17.24 \pm 9.98$ & $-1.90 \pm 6.32$ & 0.057 \\
\hline Level 5 & $-33.10 \pm 10.22$ & $-20.33 \pm 8.63$ & 0.051 & $-26.52 \pm 18.65$ & $-0.63 \pm 5.51$ & 0.100 \\
\hline Ear shift (mm) & -4.94 & -1.67 & $0.004^{*}$ & -4.61 & -2.14 & 0.700 \\
\hline
\end{tabular}

Values are presented as mean \pm standard deviation.

${ }^{*} \mathrm{p}<0.05$.

$\mathrm{mm}$ in the counter positioning group, with level 5 measuring $417.85 \mathrm{~mm}$ and $421.05 \mathrm{~mm}$, respectively. The lack of significant difference persisted after treatment with a mean head circumference of $451.94 \mathrm{~mm}$ vs. $455.30 \mathrm{~mm}$ at level 3, and $443.12 \mathrm{~mm}$ vs. $444.47 \mathrm{~mm}$ at level 5. These results suggest that neither helmet therapy nor the counter positioning interfered with head growth.

\section{DISCUSSION}

Our study reveals that helmet therapy is efficacious in terms of correcting cranial asymmetry and ear shift, while counter positioning therapy is only effective in correcting ear shift. Comparison of helmet therapy with counter positioning in patients with moderate to severe DP showed better efficacy of helmet therapy in terms of 
correcting cranial asymmetry; ear shift measurements also showed a larger degree of correction in the helmet therapy group. After reviewing both the English and the Korean literature, we believe that ours is the first report to compare the effectiveness of these 2 techniques on ear shift in patients with DP, and we believe this is the first Korean report to compare the general efficacy of these two therapeutic options in children with DP.

Conservative management, whether with helmet therapy or counter positioning, is the major therapeutic option for the treatment of DP. The superiority of helmet therapy is not yet conclusively established, due to potential biases in prior studies, such as inconsistent diagnostic criteria, varying clinical severity of DP, varying age of therapy onset, different durations of therapy, and different measurement techniques [19]. Loveday and de Chalain [18] proposed a definition of DP as a CVAI $>3.5 \%$. The clinical severity classification of DP is determined by skull asymmetry, most commonly expressed as the DD. Hutchison et al. [30] proposed a classification system, defining mild DP as a DD between $3 \mathrm{~mm}$ and $10 \mathrm{~mm}$, moderate as a DD between $10 \mathrm{~mm}$ and $12 \mathrm{~mm}$, and severe as a DD >12 $\mathrm{mm}$. Another study reported that a normal value for DD, determined by evaluating 36 healthy children without DP between the ages of 4 and 12 months, was $3 \mathrm{~mm} \pm 1 \mathrm{~mm}$ [22]. A study by Yoo et al. [25] of 108 Korean children classified DP into mild (DD between $6 \mathrm{~mm}$ and $10 \mathrm{~mm}$ ), moderate (11 $\mathrm{mm}$ to $15 \mathrm{~mm}$ ), and severe (at least $16 \mathrm{~mm}$ ).

A recent systematic review focusing on the conservative management of DP reported that favorable outcomes are 1.3 times more likely with helmet therapy than with counter positioning. However, this result is not conclusive as study biases exist regarding the clinical severity of the condition, patient age, and duration of therapy [31]. In a study by Clarren [32], all patients were offered helmet therapy, but 10 caregivers refused this treatment. Most of these caregivers had children with mild DP, resulting in more severe DP in the helmet therapy group. Graham et al. [22] compared helmet therapy with counter positioning; patients were treated with helmet therapy only after they failed to respond to counter positioning. Counter positioning has a role in correcting mild DP, but its use seems less effective in correcting moderate to severe DP. Our study analyzed only those subjects with moderate to severe DP. The results suggest that helmet therapy is superior to counter positioning in correcting cranial asymmetry in these patients. Our subgroup analysis also showed that children with more severe DP had greater improvement with helmet therapy. These results imply that helmet therapy should be considered effective treatment choice especially in severe cases of DP.

In the present study, both ear shift and cranial asymmetry showed greater improvement in the helmet therapy group than in the counter positioning group. Ear shift is one of the major cosmetic concerns in moderate to severe DP [33]. However, there are only a couple of reports in the literature investigating this phenomenon [24,34], and these provide conflicting results. Kluba et al. [24] reported that helmet therapy improves ear shift in patients who demonstrate this problem prior to treatment, but not in those who did not have this problem prior to treatment. They even reported that ear shift develops during treatment in patients who were without this problem at the beginning of treatment. Katzel et al. [34] reported that parental perception of ear shift improves after helmet therapy in their children, even though the actual measurements show no statistical difference after treatment. However, other cranial asymmetry measurements, such as CVAI and RSI do improve significantly, along with the parents' perception of the child's appearance. A prospective study by Meyer-Marcotty et al. [26], comparing the effects of helmet therapy in 20 patients with DP and 20 control subjects without the condition, showed that a comparison of measurements before and after helmet therapy showed no significant difference in ear shift, even though other cranial asymmetry measurements such as DD improved significantly. The helmet provides external force on the prominent areas of the skull, resulting in a volume shift to the flat areas in the relatively soft skull of a young child. Thus, the parallelogram-shaped cranium seen in DP can be corrected to a symmetric, oval-shaped cranium, and ear shift should also be corrected [2,14]. The present results indicate that ear shift does indeed show significant improvement with helmet therapy. Another interesting finding was that only ear shift improved to some extent with counter positioning, when other cranial asymmetry measurements showed no significant improvement in counter positioning group. Further studies should assess ear shift improvement in DP.

In this study, the head circumference in both groups of patients appeared to grow during therapy. This implies that neither helmet therapy nor counter position- 
ing interfered with head growth in children. However, we did not evaluate development in terms of cognition, language, motor function, or social functioning, all of which are important aspects in the treatment of DP. DP is often associated with congenital muscular torticollis, prematurity, or maternal multiparity, some of which are risk factors for developmental delay [1]. The fact that these may be confounding factors should be considered when comparing developmental scores between children with and without DP. In this study, we excluded children with known neurodevelopmental disorders such as cerebral palsy, autistic spectrum disorders, and genetic or metabolic diseases. Further studies should be done to examine the adverse effects of helmet therapy or counter positioning on development.

The natural course of DP and the long-term effects of helmet therapy and counter positioning are not fully understood. A large study by Boere-Boonekamp and van der Linden-Kuiper [7] in 7,609 Dutch infants revealed that $9.9 \%$ had occipital asymmetry on physical examination, and $45 \%$ of these infants had persistent asymmetry after 2 years of follow-up. A 2-year prospective cohort study of 200 infants born between 2001 and 2002 in a single hospital in New Zealand [8] reported that the prevalence of plagiocephaly and/or brachycephaly was highest at 4 months, but diminished as infants grew older. This normal progression can solve the cosmetic issues in children with mild DP, but those with moderate to severe DP may have persistent cosmetic problems despite some naturally occurring improvement. Unfortunately, there is a lack of precise data on the extent of natural improvement and the long-term effectiveness of either helmet therapy or counter positioning. To the best of our knowledge, no study has yet followed groups of children who underwent either helmet therapy or counter positioning through adulthood. A report of 28 patients treated with helmet therapy for DP and then followed for 5 years revealed that the effect of treatment appears to regress after its termination, although this regression was not statistically significant [35]. In the present study, patients were assessed immediately after the conclusion of therapy; further study is therefore needed to validate the long-term efficacy of helmet therapy versus counter positioning.

We used measurements provided by a 3D laser headsurface scanner to analyze patients' head shape. Plank et al. [27] reported that the device we used in our study provides accurate and reproducible measurements. They determined that CVAI and RSI are the most valuable measurements for assessing head shape in children with DP. However, our study has some limitations that should be considered. First, we had a small sample size, related to the strict selection criteria for study subjects. Children had to be 10 months of age or younger, with moderate to severe DP ( $\mathrm{DD} \geq 10 \mathrm{~mm}$ ). Second, the therapy group was determined by parental preference, which may have introduced bias. Third, sample size was uneven between two groups resulting in more subjects in the helmet therapy group and small subjects in the counter positioning group. Parents whose children have severe symptoms are quite possibly more likely to choose a more aggressive therapeutic option, such as helmet therapy. Although we tried to assess statistical power to ensure the statistical significance between these two small and uneven sized samples, such bias could not be completely controlled because of the retrospective nature of this study; a prospective, randomized controlled trial with a large sample of patients could help to minimize this bias. Another limitation of this study is that the compliance was assessed only based on report of the care giver. Exact compliance was not evaluated because both of therapeutic interventions used in this study were conducted in household.

In conclusion, helmet therapy in children with moderate to severe DP results in significantly more favorable outcomes in the correction of cranial and ear asymmetry than counter positioning therapy. The treatment does not appear to compromise growth of the head circumference. To the best of our knowledge, ours is the first ever report to compare the efficacy of helmet therapy and counter positioning on ear asymmetry, and the first Korean report to compare the efficacy of these 2 therapeutic options in patients with DP.

\section{CONFLICT OF INTEREST}

No potential conflict of interest relevant to this article was reported.

\section{REFERENCES}

1. Kalra R, Walker ML. Posterior plagiocephaly. Childs Nerv Syst 2012;28:1389-93.

2. Mortenson P, Steinbok P, Smith D. Deformational pla- 
giocephaly and orthotic treatment: indications and limitations. Childs Nerv Syst 2012;28:1407-12.

3. Looman WS, Flannery AB. Evidence-based care of the child with deformational plagiocephaly. Part I: assessment and diagnosis. J Pediatr Health Care 2012;26:242-50.

4. American Academy of Pediatrics Task Force on Infant Positioning and SIDS. Positioning and SIDS. Pediatrics 1992;89(6 Pt 1):1120-6.

5. Dunn PM. Congenital sternomastoid torticollis: an intrauterine postural deformity. Arch Dis Child 1974;49:824-5.

6. Roby BB, Finkelstein M, Tibesar RJ, Sidman JD. Prevalence of positional plagiocephaly in teens born after the "Back to Sleep" campaign. Otolaryngol Head Neck Surg 2012;146:823-8.

7. Boere-Boonekamp MM, van der Linden-Kuiper LT. Positional preference: prevalence in infants and follow-up after two years. Pediatrics 2001;107:339-43.

8. Hutchison BL, Hutchison LA, Thompson JM, Mitchell EA. Plagiocephaly and brachycephaly in the first two years of life: a prospective cohort study. Pediatrics 2004;114:970-80.

9. Saeed NR, Wall SA, Dhariwal DK. Management of positional plagiocephaly. Arch Dis Child 2008;93:82-4.

10. Thompson JT, David LR, Wood B, Argenta A, Simpson J, Argenta LC. Outcome analysis of helmet therapy for positional plagiocephaly using a three-dimensional surface scanning laser. J Craniofac Surg 2009;20:362-5.

11. Bialocerkowski AE, Vladusic SL, Wei Ng C. Prevalence, risk factors, and natural history of positional plagiocephaly: a systematic review. Dev Med Child Neurol 2008;50:577-86.

12. Persing J, James H, Swanson J, Kattwinkel J; American Academy of Pediatrics Committee on Practice and Ambulatory Medicine, Section on Plastic Surgery and Section on Neurological Surgery. Prevention and management of positional skull deformities in infants. Pediatrics 2003;112(1 Pt 1):199-202.

13. Clarren SK, Smith DW, Hanson JW. Helmet treatment for plagiocephaly and congenital muscular torticollis. J Pediatr 1979;94:43-6.

14. Schaaf H, Malik CY, Streckbein P, Pons-Kuehnemann J, Howaldt HP, Wilbrand JF. Three-dimensional photographic analysis of outcome after helmet treatment of a nonsynostotic cranial deformity. J Craniofac Surg
2010;21:1677-82.

15. Moss SD. Nonsurgical, nonorthotic treatment of occipital plagiocephaly: what is the natural history of the misshapen neonatal head? J Neurosurg 1997;87:667-70.

16. O’Broin ES, Allcutt D, Earley MJ. Posterior plagiocephaly: proactive conservative management. $\mathrm{Br} \mathrm{J}$ Plast Surg 1999;52:18-23.

17. Pollack IF, Losken HW, Fasick P. Diagnosis and management of posterior plagiocephaly. Pediatrics 1997;99:180-5.

18. Loveday BP, de Chalain TB. Active counterpositioning or orthotic device to treat positional plagiocephaly? J Craniofac Surg 2001;12:308-13.

19. Bialocerkowski AE, Vladusic SL, Howell SM. Conservative interventions for positional plagiocephaly: a systematic review. Dev Med Child Neurol 2005;47:563-70.

20. Katzel EB, Koltz PF, Sbitany H, Emerson C, Girotto JA. Real versus perceived improvements of helmet molding therapy for the treatment of plagiocephaly. Plast Reconstr Surg 2010;126:19e-21e.

21. Lipira AB, Gordon S, Darvann TA, Hermann NV, Van Pelt AE, Naidoo SD, et al. Helmet versus active repositioning for plagiocephaly: a three-dimensional analysis. Pediatrics 2010;126:e936-45.

22. Graham JM Jr, Gomez M, Halberg A, Earl DL, Kreutzman JT, Cui J, et al. Management of deformational plagiocephaly: repositioning versus orthotic therapy. J Pediatr 2005;146:258-62.

23. Lee A, Van Pelt AE, Kane AA, Pilgram TK, Govier DP, Woo AS, et al. Comparison of perceptions and treatment practices between neurosurgeons and plastic surgeons for infants with deformational plagiocephaly. J Neurosurg Pediatr 2010;5:368-74.

24. Kluba S, Schreiber R, Kraut W, Meisner C, Reinert S, Krimmel M. Does helmet therapy influence the ear shift in positional plagiocephaly? J Craniofac Surg 2012;23:1301-5.

25. Yoo HS, Rah DK, Kim YO. Outcome analysis of cranial molding therapy in nonsynostotic plagiocephaly. Arch Plast Surg 2012;39:338-44.

26. Meyer-Marcotty P, Bohm H, Linz C, Kunz F, Keil N, Stellzig-Eisenhauer A, et al. Head orthesis therapy in infants with unilateral positional plagiocephaly: an interdisciplinary approach to broadening the range of orthodontic treatment. J Orofac Orthop 2012;73:151-65. 27. Plank LH, Giavedoni B, Lombardo JR, Geil MD, Reis- 
ner A. Comparison of infant head shape changes in deformational plagiocephaly following treatment with a cranial remolding orthosis using a noninvasive laser shape digitizer. J Craniofac Surg 2006;17:1084-91.

28. Faul F, Erdfelder E, Lang AG, Buchner A. G*Power 3: a flexible statistical power analysis program for the social, behavioral, and biomedical sciences. Behav Res Methods 2007;39:175-91.

29. Faul F, Erdfelder E, Buchner A, Lang AG. Statistical power analyses using $\mathrm{G}^{*}$ Power 3.1: tests for correlation and regression analyses. Behav Res Methods 2009;41:1149-60.

30. Hutchison BL, Hutchison LA, Thompson JM, Mitchell EA. Quantification of plagiocephaly and brachycephaly in infants using a digital photographic technique. Cleft Palate Craniofac J 2005;42:539-47.

31. Xia JJ, Kennedy KA, Teichgraeber JF, Wu KQ, Baumgartner JB, Gateno J. Nonsurgical treatment of defor- mational plagiocephaly: a systematic review. Arch Pediatr Adolesc Med 2008;162:719-27.

32. Clarren SK. Plagiocephaly and torticollis: etiology, natural history, and helmet treatment. J Pediatr 1981;98:92-5.

33. Argenta L, David L, Thompson J. Clinical classification of positional plagiocephaly. J Craniofac Surg 2004;15:368-72.

34. Katzel EB, Koltz PF, Sbitany H, Girotto JA. Treatment of plagiocephaly with helmet molding therapy: do actual results mimic perception? Cleft Palate Craniofac J 2011;48:205-9.

35. Lee RP, Teichgraeber JF, Baumgartner JE, Waller AL, English JD, Lasky RE, et al. Long-term treatment effectiveness of molding helmet therapy in the correction of posterior deformational plagiocephaly: a fiveyear follow-up. Cleft Palate Craniofac J 2008;45:240-5. 